\title{
Phonon thermodynamics and elastic behavior of GaAs at high temperatures and pressures
}

\author{
Jane E. Herriman $\oplus^{1,2}$ and Brent Fultz $\oplus^{1}$ \\ ${ }^{1}$ Department of Applied Physics and Materials Science, California Institute of Technology, Pasadena, California 91125, USA \\ ${ }^{2}$ Quantum Simulations Group, Lawrence Livermore National Laboratory, Livermore, California 94550, USA
}

(Received 4 January 2020; revised manuscript received 12 April 2020; accepted 27 May 2020;

published 11 June 2020)

\begin{abstract}
The phonons of wurtzite and zinc blende GaAs were calculated at simultaneously elevated temperature and pressure, and elastic constants were calculated as functions of pressure. Pressure caused instabilities of shorterwavelength transverse acoustic modes in both wurtzite and zinc blende GaAs, causing them to fall to zero at 18 and $20 \mathrm{GPa}$, respectively. The Born stability criteria, which depend on elastic constants and only long wavelength phonons, therefore overestimated the pressure needed to induce instability at $0 \mathrm{~K}$. At elevated temperatures, explicit anharmonicity pushes the onset of instability to higher pressures in both wurtzite and zinc blende GaAs. Phonon linewidth and densities of states data showed that the quasiharmonic approximation failed to account for temperature-induced phonon frequency shifts, and the quasiharmonic approximation became less reliable at elevated pressure. In general, the number of three-phonon processes increased with pressure, thereby increasing the temperature-driven broadening of phonon spectral lineshapes.
\end{abstract}

DOI: 10.1103/PhysRevB.101.214108

\section{INTRODUCTION}

Gallium arsenide, GaAs, is a well-studied semiconductor with important technological applications in transistors [2-4], photovoltaic devices [5], and photon detectors [6]. Although less earth-abundant and more expensive than silicon, GaAs offers several advantages. It has a higher electron mobility than silicon, allowing for devices with higher operating frequencies. Today, GaAs dominates the circuitry in critical mobile phone components, such as power amplifiers [7]. Unlike $\mathrm{Si}$, GaAs has a direct band gap that suppresses phonon creation during photon emission, enabling its use in light emitting diodes (LEDs) [8], laser diodes [9], and optical communications [10]. The band gap of GaAs is wider than that of $\mathrm{Si}$, making it more resistant to radiation damage, and a more attractive material for satellites [5] and deep space electronics [3].

Like many III-V materials, GaAs is polytypic [11,12]. It has the zinc blende crystal structure under ambient conditions, but the wurtzite phase is metastable and has been observed experimentally [12]. Both zinc blende and wurtzite crystal structures consist of two interpenetrating face-centered cubic (fcc) or hexagonal close-packed (hcp) sublattices, separately populated by Ga and As atoms (Fig. 1). High pressure induces a structural phase transition in zinc blende GaAs to an orthorhombic structure between $12 \mathrm{GPa}$ [13] and $17 \mathrm{GPa}$ [14].

Modeling thermophysical properties of materials is an important and active part of materials physics and materials engineering. Modeling can enable explorations of extreme conditions, including extreme pressures and temperatures, and quick transversals of parameter spaces. The temperature and pressure evolution of phonon spectra and elastic properties are of interest because they are closely related to the Gibbs free energy and crystal stability. Phonon spectra also offer a microscopic probe into thermal conductivity, thermal expansion, and the temperature dependence of elastic constants. The widely used quasiharmonic approximation (QHA) extends the harmonic approximation by including effects of volume on phonon frequencies. The QHA ignores explicit anharmonic effects from the anharmonic potentials in which atoms vibrate, where displacements from their equilibrium positions cause forces that do not obey Hooke's law. These anharmonic effects increase with temperature, and can prevent the QHA from correctly modeling thermal expansion [15] or phase stability [16].

Here we report elastic and phonon properties of wurtzite and zinc blende GaAs at simultaneously elevated temperature and pressure. From the elastic constants, we predict changes with pressure of the elastic stability, elastic anisotropy (see Supplemental Material [17]), and anharmonic effects on phonon frequencies and linewidths. Our methods are described in Sec. II, results in Sec. III, and the context for, and implications of, these results are given in Sec. IV. Most notably, we report that the lattice stabilities of both wurtzite and zinc blende GaAs are not predicted by the Born stability criteria because the early onset of shorter wavelength phonon instabilities precede elastic collapse. These instabilities are shown to change with temperature owing to effects of phonon anharmonicity. We also report a large effect of pressure on phonon anharmonicity.

\section{COMPUTATIONAL}

Projector augmented wave (PAW) potentials and the local density approximation (LDA) were used in the Vienna $A b$ initio Simulation Package (VASP) [18,19], an implementation of density functional theory (DFT) [20], to perform firstprinciples calculations on GaAs. All calculations used an energy cutoff of $600 \mathrm{eV}$ and a minimum of 13,824 $k$-points $\times$ 

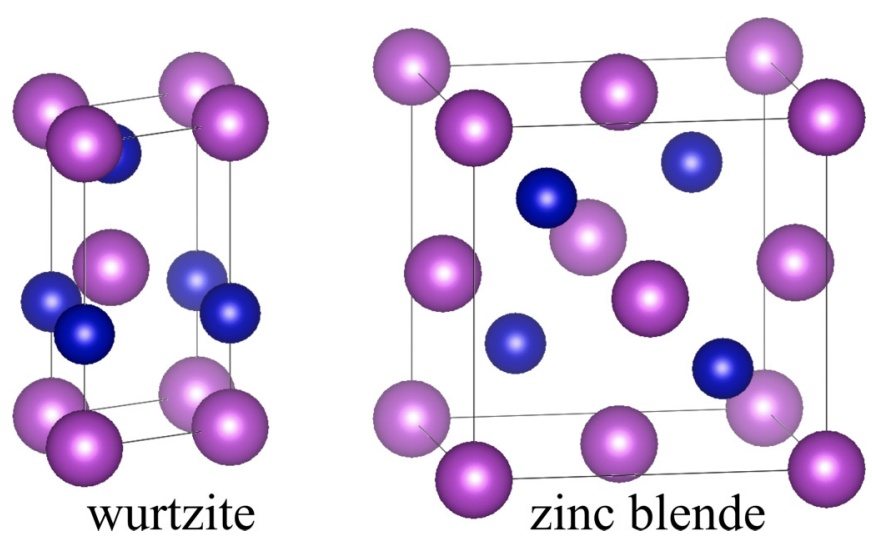

FIG. 1. Unit cells of wurtzite (left) and zinc blende (right) GaAs [1]. This wurtzite unit cell is primitive; the larger, nonprimitive unit cell for zinc blende more clearly shows its cubicity.

atoms. The work required calculations for both unit cells and supercells. In wurtzite GaAs, four atom unit (primitive) cells were used with a $20 \times 20 \times 12 k$-point mesh, and supercells of 192 atoms used a $5 \times 5 \times 4 k$-point mesh. In zinc blende GaAs, unit cells with two atoms unit (primitive) cells were used with a $22 \times 22 \times 22 k$-point mesh and supercells with 216 atoms used a $4 \times 4 \times 4 k$-point mesh. The supercells had approximately the same lengths across all three dimensions. $k$-point meshes were generated with a Monkhorst-Pack [21] scheme.

For each material, the total energies of static unit cells were calculated with relaxed shapes and ionic positions of varying size, with volumes scaled between approximately $69 \%$ and $108 \%$ of the equilibrium volume. The resulting energyvolume relationships were used with a Birch-Murnaghan equation of state, allowing the determination of pressure for any volume within this range. The elastic constants for unit cells held at pressures between about -5 and $65 \mathrm{GPa}$ in $0.5 \mathrm{GPa}$ steps were calculated, using stress-strain calculations in VASP.

With supercells, the phonon properties (phonon dispersion relations, densities of states, and linewidths) of each material were calculated at 0,600 , and $1200 \mathrm{~K}$ for pressures of $0.0,5.0$, 7.5, 10.0, and 15.0 GPa, accounting for both quasiharmonic effects of volume and anharmonic effects of temperature. Separately, phonon properties for these temperatures and pressures were calculated quasiharmonically by ignoring the effects of finite temperature except for thermal expansion, and such calculations are labeled "QHA." (Quasiharmonic volumes at each pressure and temperature of interest were equal to those used for corresponding calculations including anharmonic phonon effects.) At each temperature, pressurevolume relationships and selected volumes corresponding to each pressure of interest were determined by fitting a BirchMurnaghan equation of state to Helmholtz free energy versus volume trends of that isotherm.

Helmholtz free energies, $F(V, T)$, were calculated by summing the ground-state total energy surface, $E_{0}(V)$ (derived from the energy-volume relationships from static unit cells), and the temperature-dependent phonon free energies, calculated at several volumes for a given temperature

$$
F(V, T)=E_{0}(V)+F_{\mathrm{ph}}(V, T) .
$$

We calculated finite temperature phonon properties using the temperature-dependent effective potential (TDEP) method [22-24]. For an ensemble of supercells with thermal displacement patterns and their interatomic forces as calculated by VASP, TDEP generates a model potential energy as

$$
U=U_{0}+\frac{1}{2 !} \sum_{i j \alpha \beta} \phi_{i j}^{\alpha \beta} u_{i}^{\alpha} u_{j}^{\beta}+\frac{1}{3 !} \sum_{i j k \alpha \beta \gamma} \phi_{i j k}^{\alpha \beta \gamma} u_{i}^{\alpha} u_{j}^{\beta} u_{k}^{\gamma} .
$$

The TDEP method optimizes $U_{0}$ and $\phi_{i j}^{\alpha \beta}$ and $\phi_{i j k}^{\alpha \beta \gamma}$, which are interatomic force constants for two- and three-body interactions. These model parameters are chosen to minimize the difference between the set of interatomic forces determined by VASP and those predicted by the model potential of Eq. (2). The TDEP calculations used Born effective charge tensors from VASP with the correction scheme of Gonze and Lee $[25,26]$ to account for long-range interactions in polar GaAs.

A stochastic sampling method (s-TDEP) [27] was used to generate an ensemble of (typically 20 to 40, depending on structural symmetries and temperature) supercells with thermal atomic displacements. The scheme generates position vectors for the $i$ th atom, $\left\{\mathbf{u}_{i}\right\}$, consistent with phonon displacements and Bose-Einstein statistics

$$
\mathbf{u}_{i}=\sum_{s=1}^{3 N_{a}} \epsilon_{i s}\left\langle A_{i s}\right\rangle \sqrt{-2 \ln \xi_{1}} \sin 2 \pi \xi_{2}
$$

where $\left\{\epsilon_{i s}\right\}$ are the eigenvectors corresponding to the $\{s\}$ phonon modes in a system of $N_{a}$ atoms. The embedded expression $\sqrt{-2 \ln \xi_{1}} \sin 2 \pi \xi_{2}$ is a Box-Muller transform generating a random variable with a standard normal distribution, given two uniformly distributed random numbers, $\xi_{n}$, between 0 and 1 . Finally, $\left\langle A_{i s}\right\rangle[28,29]$ is the temperature-dependent expectation value of the normal mode amplitude of the $s$ th phonon mode of the $i$ th atom, given by

$$
\left\langle A_{i s}\right\rangle=\sqrt{\frac{\hbar\left(2 n_{s}+1\right)}{2 m_{i} \omega_{s}}}=\sqrt{\frac{\hbar\left(\frac{2}{e^{\frac{\hbar \omega_{S}}{B_{T}}}-1}+1\right)}{2 m_{i} \omega_{s}}},
$$

which in the classical limit becomes

$$
\left\langle A_{i s}\right\rangle \approx \frac{1}{\omega_{s}} \sqrt{\frac{k_{B} T}{m_{i}}},
$$

where $\omega_{s}$ and $n_{s}$ are the eigenvalue and thermal occupation of the $s$ th phonon mode and $m_{i}$ is the mass of the $i$ th atom.

Each atomic displacement, $\mathbf{u}_{i}$, is therefore a thermallyweighted superposition of all vibrational modes, with randomness injected to simulate sampling atomic positions at different times. Zero-point contributions are included with Eq. (4) [30]. Generating these atomic positions requires prior knowledge of the phonon mode eigenvectors and eigenvalues, accessible given interatomic force constants. We therefore began by seeding these calculations with force constants either from frozen phonon calculations or pre-existing work on similar systems; phonon calculations consisting of stochastically generated ensembles were then performed for two to four iterations until the interatomic force constants converged. 


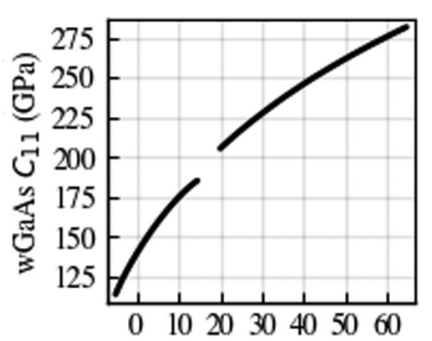

(a)

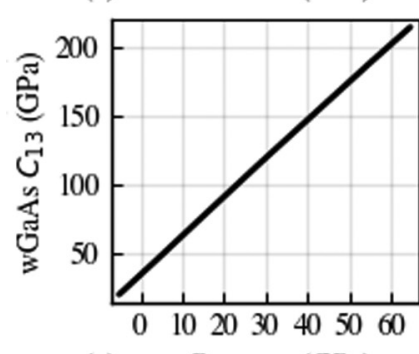

(c) Pressure (GPa)

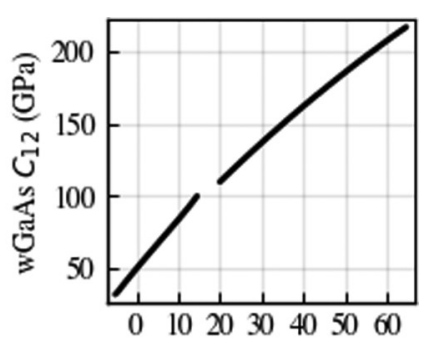

(b)

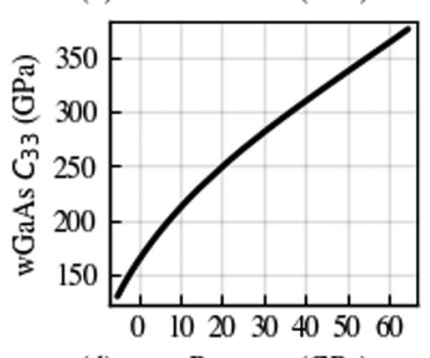

(d) Pressure (GPa)

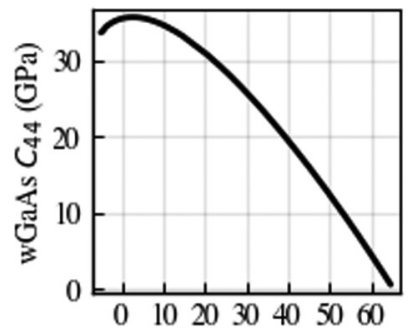

(e)

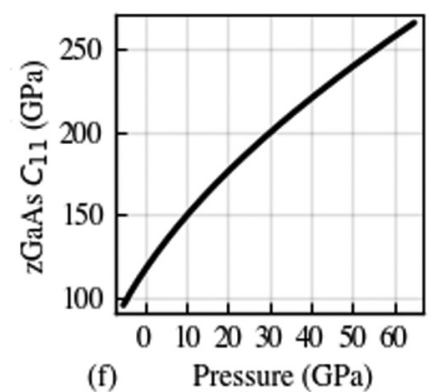

Pressure (GPa)
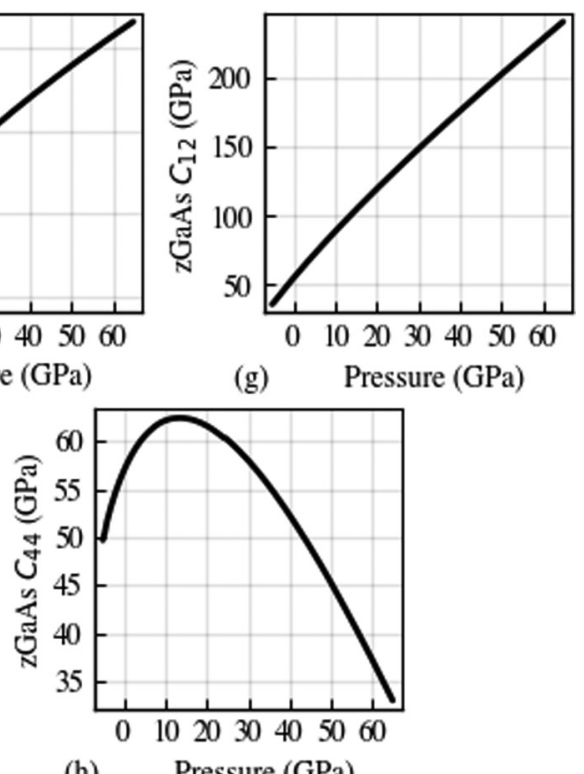

(h)

Pressure (GPa)

FIG. 2. Elastic moduli in GPa plotted versus pressure, also in GPa, for (a)-(e) wGaAs and for (f)-(h) zGaAs. For $C_{11}$ and $C_{12}$ in wGaAs, we omitted data from approximately 15 to $20 \mathrm{GPa}$, where evidence of a phonon instability emerges, as shown in Fig. 4, to artificially decouple results on the elastic properties from this instability.

\section{RESULTS}

Figure 2 presents elastic constants for wGaAs [Figs. 2(a) to 2(e)] and zGaAs [Figs. 2(f) to 2(h)] as functions of pressure.

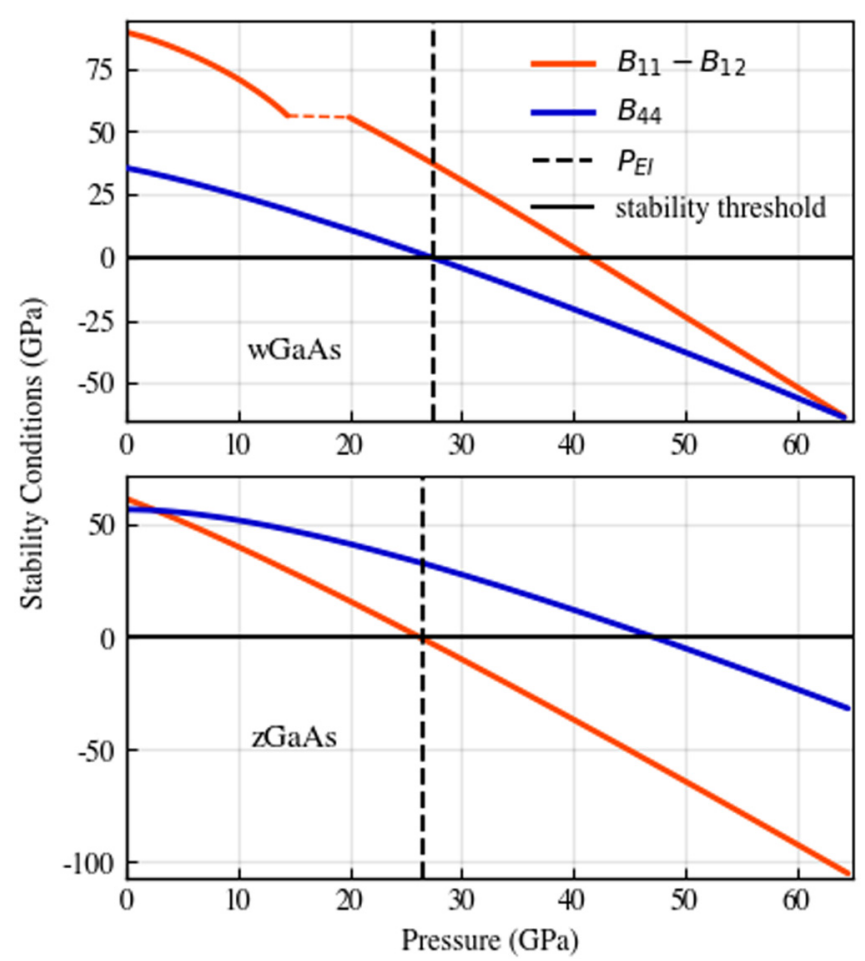

FIG. 3. Illustration of the first two Born stability criteria versus pressure for wGaAs (top) and zGaAs (bottom). The dashed orange line is a guide to the eye spanning the region where data have been omitted from Fig. 2 for $C_{11}$ and $C_{12}$. A dashed vertical black line at $27 \mathrm{GPa}$, labeled $P_{E I}$, indicates the pressure at which elastic instability occurs in each phase.

These results were obtained from VASP without accounting for any temperature effects, i.e., from relaxed unit cells with volumes at each pressure determined by a Birch-Murnaghan equation of state fit to energy-volume relationships that did not account for any phonon free energy. The elastic constants for both materials increase with pressure, except for $C_{44}$, which decreases.

We do not show values for $C_{11}$ and $C_{12}$ between about 15 and $20 \mathrm{GPa}$ in wGaAs, for reasons that will be explained below using Figs. 4 and 5. Figure 3 presents results pertaining to elastic stability that derive from the elastic constants shown in Fig. 2. As for $C_{11}$ and $C_{12}$ in Fig. 2, we do not plot real data from about 15 to $20 \mathrm{GPa}$ in Fig. 3.

We predicted when wGaAs and zGaAs would become elastically unstable using Born stability criteria that account for pressure [31-34]. The three stability conditions that must be met for cubic crystals such as zinc blende are

$$
B_{11}-B_{12}>0, B_{44}>0, B_{11}+2 B_{12}>0,
$$

and for hexagonal crystals such as wurtzite

$$
B_{11}-\left|B_{12}\right|>0, B_{44}>0, B_{33}\left(B_{11}+B_{12}\right)>2 B_{13}^{2} .
$$

The elastic stiffnesses $B_{i j}$ are the corresponding values of elastic constants $C_{i j}$ with pressure

$$
\begin{gathered}
B_{i i}=C_{i i}-P, i=1,2, \ldots, 6, \\
B_{1 j}=C_{1 j}+P, j=2,3 .
\end{gathered}
$$



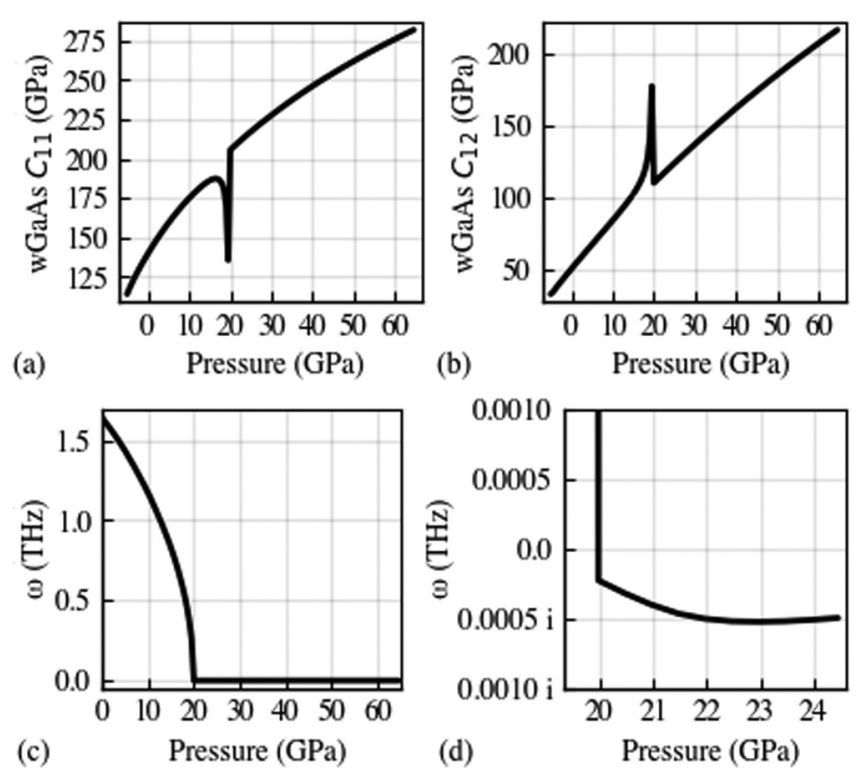

FIG. 4. In panels (a) and (b), $C_{11}$ and $C_{12}$ are plotted against pressure for wGaAs without omitting data from 15 to $20 \mathrm{GPa}$ as done in Fig. 2. The respective dip and spike shown correspond to contributions to these elastic moduli that scale roughly inversely with certain $\Gamma$-point phonon frequencies as they approach zero. Below, in (c) and (d), frequencies for the degenerate transverse optical phonon modes at $\Gamma$ are plotted against pressure. Panel (c) shows that these phonon modes soften considerably until approaching $0 \mathrm{THz}$ at $20 \mathrm{GPa}$. In (d) an expanded plot shows us that at $20 \mathrm{GPa}$ frequencies for this phonon mode become imaginary, giving the onset of a phonon instability.

The third stability conditions of Eqs. (6) and (7) remain true across our entire pressure range of 0 to $65 \mathrm{GPa}$ for both wGaAs and zGaAs. Figure 3 shows the first two Born instabilities in wGaAs and zGaAs. The first stability condition fails when $B_{11}-B_{12}\left(B_{11}-\left|B_{12}\right|\right.$ for wurtzite) falls to zero. Similarly, the second stability condition fails when $B_{44}$ crosses zero. According to the Born stability criteria, both $w G a A s$ and zGaAs become unstable at $27 \mathrm{GPa}$, denoted by the vertical dashed lines. For wGaAs this occurs when $B_{44}$ falls to zero; for zGaAs there is a "tetragonal shear instability"[33] that occurs when $B_{12}$ becomes greater than $B_{11}$.

Finally we address the omitted data from 15 to $20 \mathrm{GPa}$ in the trends for $C_{11}$ and $C_{12}$ of wGaAs in Fig. 2, and phonon phenomena in this range of pressure. Figures 4(a) and 4(b) show the full pressure trends for $C_{11}$ and $C_{12}$ of wGaAs. Between 15 to $20 \mathrm{GPa}, C_{11}$ exhibits an anomalous dip; simultaneously $C_{12}$ exhibits an anomalous spike. While the observed dip and spike are themselves nonphysical, they reflect a phonon instability. The dip and spike are side effects of the way VASP calculates elastic constants via stress-strain relationships. VASP distorts the crystal unit cell and obtains the elastic constants from the resulting stress-strain relationships. The output is a version of the elastic tensor containing symmetrized elastic moduli. However, these symmetrized elastic moduli do not account for the fact that the ionic positions of the unit cell are no longer relaxed once each distortion is applied. To correct for this, VASP calculates the contribution to the elastic tensor from

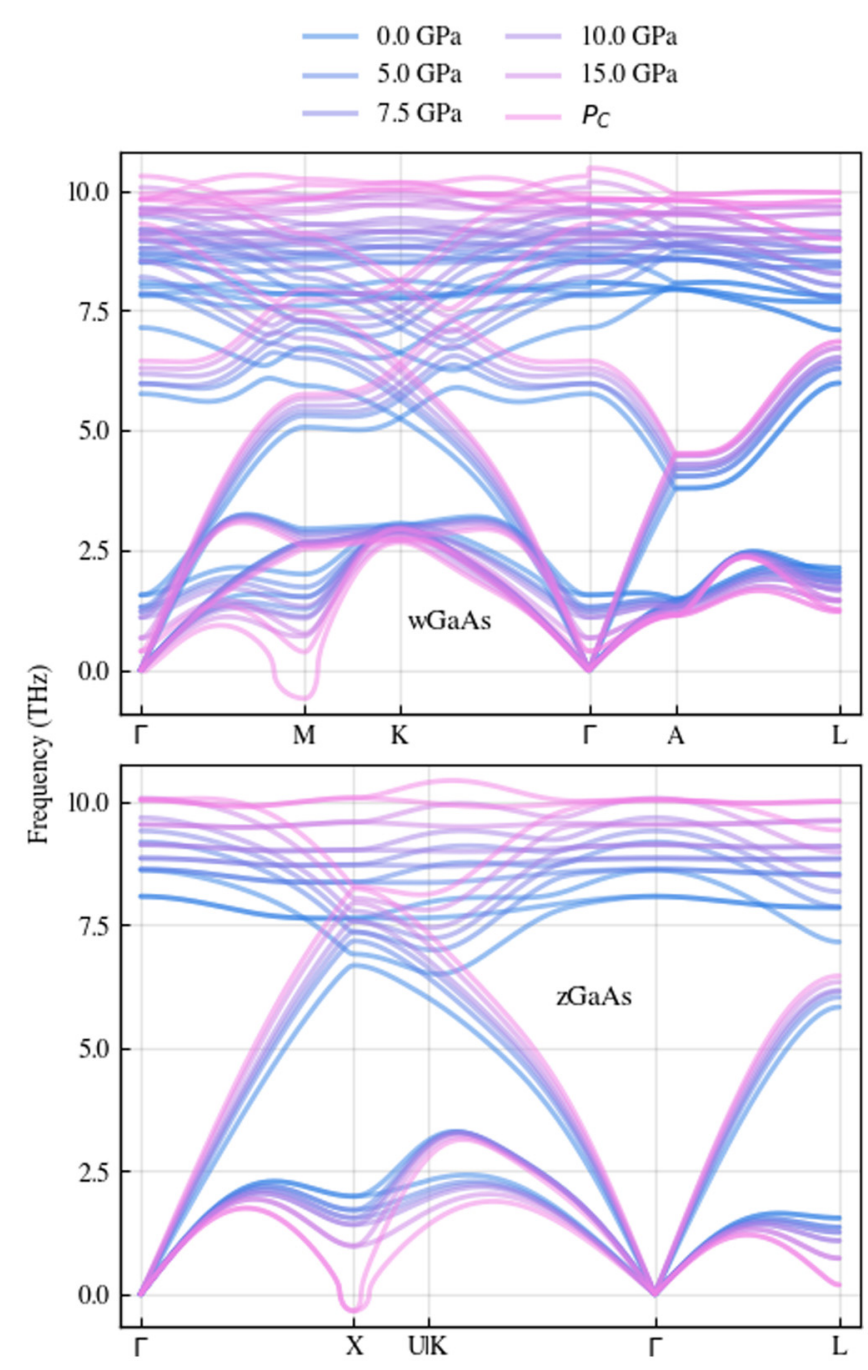

FIG. 5. Phonon dispersion relations at $0 \mathrm{~K}$ for wGaAs (top) and zGaAs (bottom) with frequencies on the vertical axis plotted against phonon wave vectors along high symmetry directions. Phonon dispersion relations at various pressures are overlaid, with increasing pressure from blue to purple to pink. In wGaAs, we see pressureinduced softening of the longitudinal acoustic mode at $M$ and of the degenerate transverse optical modes at $\Gamma$, until an instability occurs around $18 \mathrm{GPa}$ at $M$, denoted by $P_{C}$. In zGaAs, the transverse acoustic modes soften at $X$ and $L$ with increasing pressure, until a phonon instability occurs around $20 \mathrm{GPa}$ at $X$.

ionic relaxation and adds this contribution to the symmetrized elastic moduli, giving the total elastic moduli. VASP calculates the contributions from the ionic relaxation using a dynamical matrix constructed from phonon frequencies at the $\Gamma$ point. In wGaAs, the dip in $C_{11}$ and spike in $C_{12}$ correspond to the contributions to elastic moduli from ionic relaxation. These contributions to the ionic relaxation are made nonphysical by a phonon instability as seen in Figs. 4(c) and 4(d), where the phonon frequencies for the lowest-energy transverse optical modes at $\Gamma$ become imaginary at $20 \mathrm{GPa}$.

More direct evidence of phonon instabilities occurring at pressures below the Born instabilities is seen in Fig. 5, where phonon dispersions are shown for wGaAs and zGaAs. In 


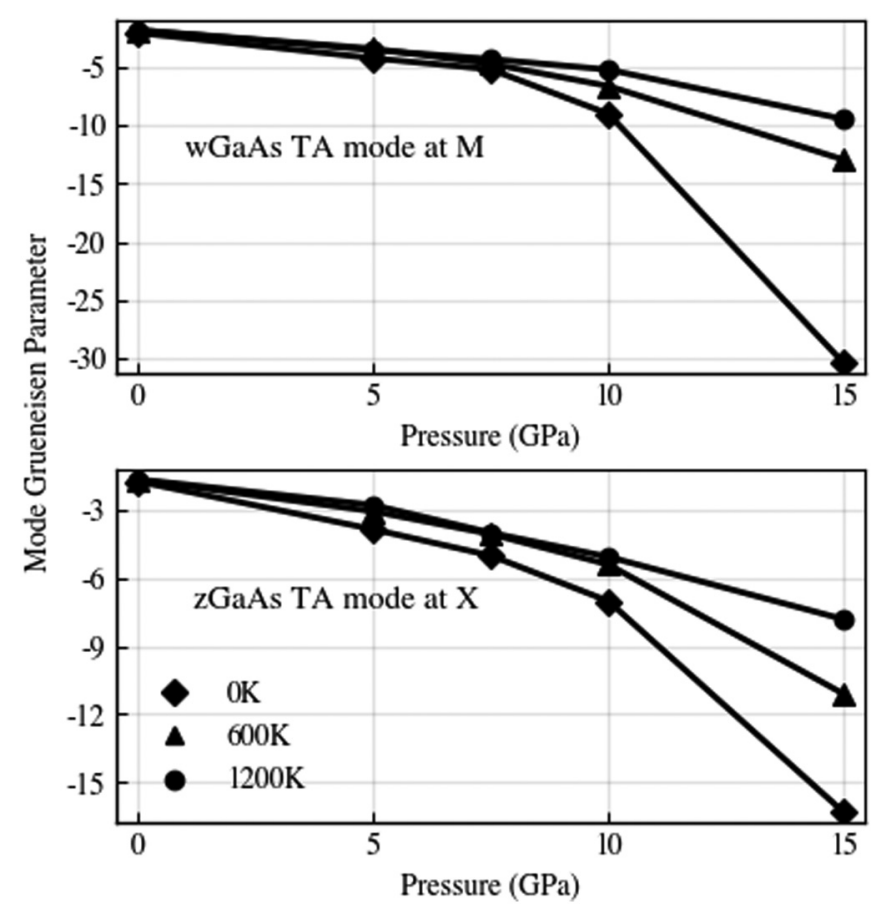

FIG. 6. Mode Grüneisen parameters that are the most sensitive to volume changes are plotted against pressure for 0,600 , and $1200 \mathrm{~K}$ in wGaAs (top) and zGaAs (bottom).

wGaAs we see significant phonon softening with pressure, not only for the lowest frequency transverse optical modes at $\Gamma$, but also from the transverse acoustic mode at $M$. At both these phonon wave vectors, the modes soften until their frequencies go imaginary. Figure 5 presents the onset of phonon instability at the $M$ point only, showing that it occurs at a lower pressure than the phonon instability at $\Gamma$. The first phonon instability in wGaAs occurs at about $18 \mathrm{GPa}$. Similarly, in zGaAs there is a large softening of transverse acoustic modes at $X$ and at $L$. In zGaAs the first phonon instability occurs at $X$, near $20 \mathrm{GPa}$. Because zGaAs exhibits no phonon instability at $\Gamma$ and only $\Gamma$ point frequencies contribute to determinations of the elastic constants in VASP, no strange discontinuities are observed in the elastic constants of zGaAs in Fig. 2.

The temperature dependence of the Grüneisen parameters of the unstable phonon modes, calculated from third order force constants in TDEP [23], are shown in Fig. 6. At 0, 600, and $1200 \mathrm{~K}$, the Grüneisen parameters for the lowest-energy transverse acoustic modes at the $M$ point in wGaAs and at $X$ in zGaAs are negative. They become increasingly negative with pressure, meaning that their frequencies become more sensitive to pressure, accelerating the onset of instability. At higher temperatures, the Grüneisen parameters of these modes are smaller in magnitude, and change less rapidly with increasing pressure. Because the pressure sensitivities of these phonon frequencies decrease with temperature, the onsets of the phonon instabilities are shifted to higher pressures at higher temperatures.

Figure 7 shows the phonon densities of states for wGaAs and zGaAs. Each panel shows the phonon DOS at $0 \mathrm{~K}$, at $1200 \mathrm{~K}$ from the QHA, and at $1200 \mathrm{~K}$ with both quasiharmonic and explicitly anharmonic effects. At $0 \mathrm{GPa}$, the

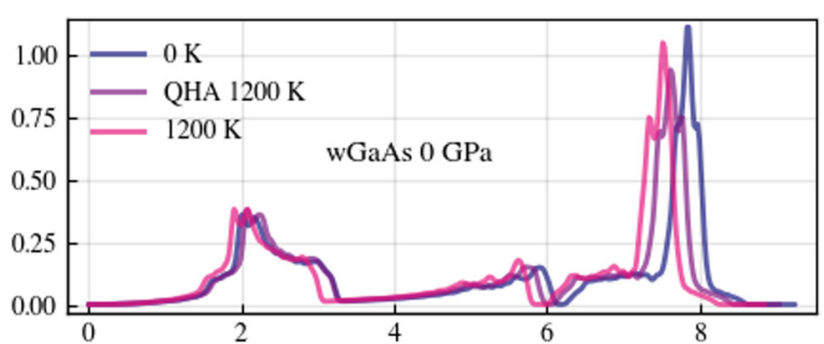

(a)

Frequency $(\mathrm{THz})$

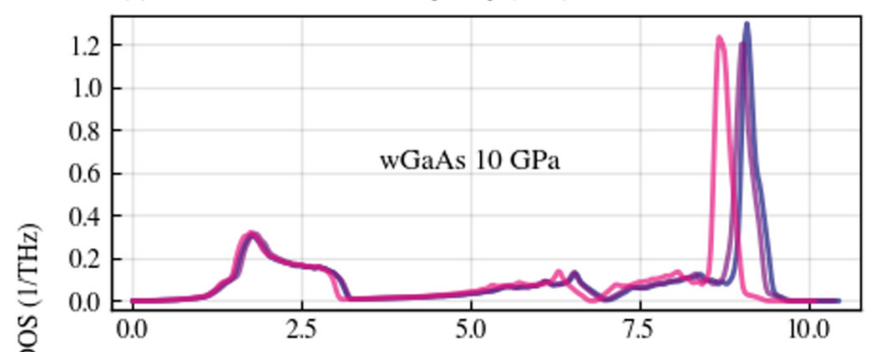

(b) Frequency $(\mathrm{THz})$

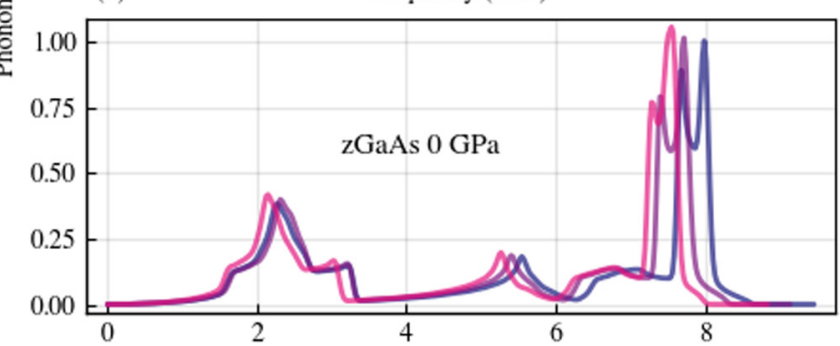

(c)

Frequency $(\mathrm{THz})$

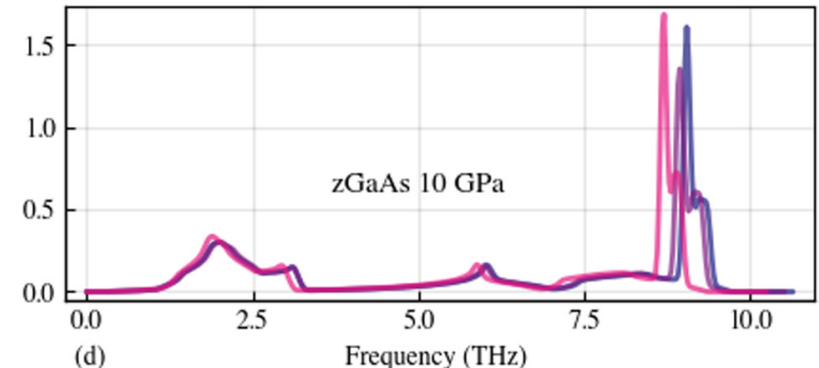

FIG. 7. Overlays of phonon DOS at $0 \mathrm{~K}$ and at $1200 \mathrm{~K}$ per a QHA and with full anharmonic phonon effects are shown at (a) $0.0 \mathrm{GPa}$ in wGaAs, (b) $10.0 \mathrm{GPa}$ in wGaAs, (c) $0.0 \mathrm{GPa}$ in zGaAs, and (d) $10.0 \mathrm{GPa}$ in zGaAs. At elevated pressures, the QHA is less reliable for predicting thermal shifts.

quasiharmonic phonon DOS at $1200 \mathrm{~K}$ is more similar to the phonon DOS at $1200 \mathrm{~K}$ with explicit anharmonicity than it is to the phonon DOS at $0 \mathrm{~K}$. At $10 \mathrm{GPa}$, this is no longer true and the quasiharmonic phonon DOS at $1200 \mathrm{~K}$ are much more similar to results at $0 \mathrm{~K}$ than at $1200 \mathrm{~K}$. At elevated pressure, the QHA becomes less effective at approximating the effects of temperature.

Figure 8 shows that the effects of pressure on thermal phonon linewidths are mode-dependent. Here we overlay phonon dispersions for wGaAs and zGaAs, respectively, with pressure- and temperature-dependent broadening at $1200 \mathrm{~K}$ and 0 and $10 \mathrm{GPa}$. The thicknesses of the phonon branches are the phonon linewidths, i.e., the imaginary component of the phonon self energy as calculated in TDEP [23]. (The 


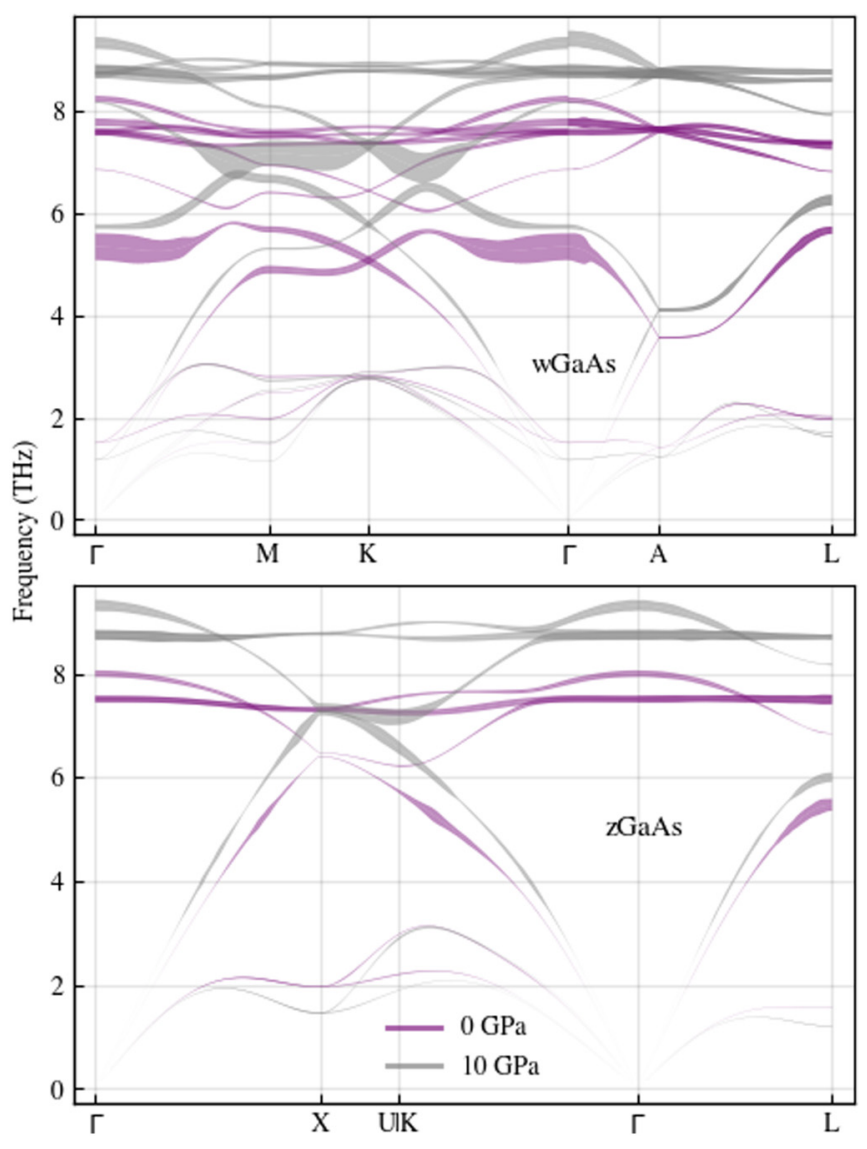

FIG. 8. Calculated spectral weights showing phonon dispersions for wGaAs (top) and zGaAs (bottom), showing broadening from phonon-phonon processes at $1200 \mathrm{~K}$ for $0.0 \mathrm{GPa}$ (purple) and $10.0 \mathrm{GPa}$ (gray).

linewidths are nearly $0 \mathrm{THz}$ at $0 \mathrm{~K}$ in both materials.) The purple and gray lines in each panel show the broadening at $1200 \mathrm{~K}$ for $0 \mathrm{GPa}$ and at $1200 \mathrm{~K}$ for $10 \mathrm{GPa}$, respectively. Increasing the pressure at high temperature causes the spectral shape of some phonon modes to broaden in energy, and others to narrow. In wGaAs, for example, the broadening of modes at $\Gamma$ near $5 \mathrm{THz}$ decreases with pressure, whereas the broadening of modes at $A$ near $4 \mathrm{THz}$ increases with pressure. Similarly in zGaAs, pressure can cause broadening to increase, as at $X$ near $7 \mathrm{GPa}$, or to decrease, as at $L$ near $5 \mathrm{THz}$.

Figure 9 compares the average effect of pressure on phonon broadening in wGaAs and zGaAs to that in wGaN and zGaN, using data from our previous work [35]. The graph shows the ratio of the values between high pressure and low pressure of the average phonon linewidths. The average linewidth was from all phonon branches across the high symmetry paths in the dispersion curves of Fig. 8. In wGaAs and zGaAs, our high and low pressures are $10 \mathrm{GPa}$ and $0 \mathrm{GPa}$; in wGaN and zGaN they are $30 \mathrm{GPa}$ and $0 \mathrm{GPa}$. For both wGaAs and zGaAs, this ratio is greater than 1 for 0,600 , and $1200 \mathrm{~K}$ and modestly decreases with temperature, indicating a temperature-dependent increase in broadening with increasing pressure. Conversely in both $\mathrm{wGaN}$ and $\mathrm{zGaN}$, this ratio is smaller than 1 at both 0 and $1120 \mathrm{~K}$, indicating a decrease in thermal phonon broadening with increasing pressure.

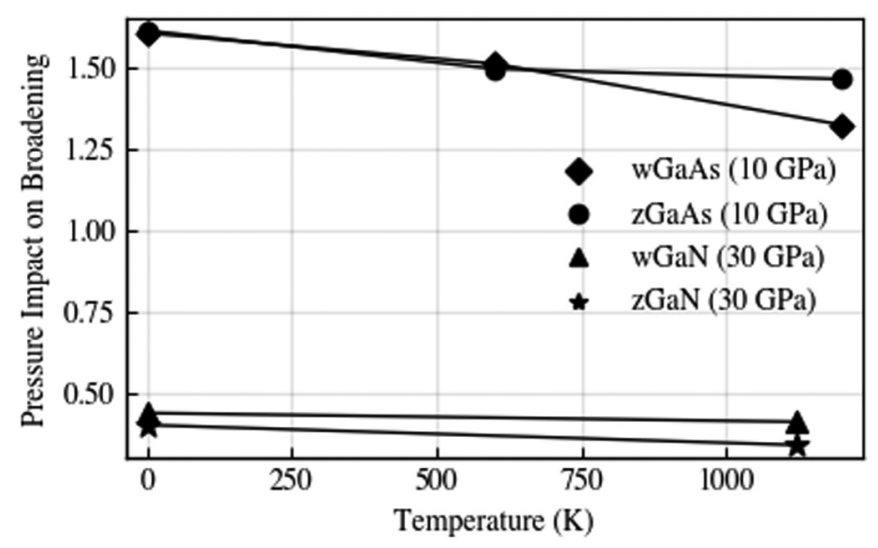

FIG. 9. The factor by which average linewidth across high symmetry paths for all phonon branches changes with a pressure of either $10 \mathrm{GPa}(\mathrm{GaAs})$ or $30 \mathrm{GPa}(\mathrm{GaN})$ [35] versus temperature for wGaAs, zGaAs, wGaN, and zGaN. Values greater than 1 show that pressure increases broadening. Values less than 1 indicate that pressure decreases broadening.

\section{DISCUSSION}

\section{A. Elastic properties and origin of lattice instability}

The Born stability criteria implicitly account for the behavior of long-wavelength acoustic phonon modes near $\Gamma$, but assume the stability of shorter-wavelength phonon modes. There is a correspondence between elastic constants and interatomic force constants in the long wavelength limit. As explained in other work [36,37], the elastic constants can be used to construct an analog to the dynamical matrix, $\mathbf{A}(\mathbf{q})$

$$
A_{i j}(\mathbf{q})=\sum_{k l} C_{i k j l} q_{k} q_{l},
$$

where $\mathbf{q}$ is a point in reciprocal space and $C_{i k j l}$ is an element of the elastic tensor, equivalent in Voigt notation to $C_{i j}$. The linear eigenvalue equation

$$
\mathbf{A}(\mathbf{q}) \mathbf{u}(\mathbf{q})=\rho \omega^{2}(\mathbf{q}) \mathbf{u}(\mathbf{q}),
$$

holds near $\mathbf{q}=0$, giving frequencies of long wavelength phonon modes, scaled by the density $\rho$, where $\mathbf{u}(\mathbf{q})$ is the corresponding eigenvector at $\mathbf{q}$.

In wGaAs a phonon instability occurs near the $\Gamma$-point. It is revealed by imaginary frequencies in Eq. (11), and gives a strange dip and spike in $C_{11}$ and $C_{12}$ at $20 \mathrm{GPa}$ when VASP calculates elastic constants. Nevertheless, these transverse acoustic modes at $\Gamma$ are not the first to become unstable in wGaAs. The first instability is at $18 \mathrm{GPa}$ in the transverse acoustic modes at $M$. This is evident in the phonon dispersions in Fig. 5, but not in the elastic constants of Figs. 2 or 3 (because VASP reports and uses $\Gamma$ frequencies but not others to calculate $C_{i j}$ ). This phonon instability in wGaAs at $18 \mathrm{GPa}$ is approximately $9 \mathrm{GPa}$ below the Born instability at $27 \mathrm{GPa}$. In zGaAs, the first instability occurs at $20 \mathrm{GPa}$ for a transverse acoustic mode at $X$. By examining the eigenvectors of the unstable transverse mode at $M$ in wGaAs, we found that it has atom displacements in the basal plane, perpendicular to the $z$-direction. Likewise, the unstable transverse acoustic mode in zGaAs at $L$ has atom displacements in its close-packed 
$\{111\}$ planes. Other reports that short wavelength modes can become unstable before the onset of Born instabilities include Refs. [38,39], and an older analysis with Morse potentials predicted that this would cause amorphization of GaAs [40].

It is interesting to compare these results on GaAs to the elastic properties of wurtzite and zinc blende gallium nitride, GaN. In contrast to wGaAs and zGaAs, wGaN and zGaN did not exhibit phonon instabilities prior to the onset of Born instabilities. The phonon modes that soften to zero in GaAs under pressure show only a small tendency towards softening for both $w \mathrm{GaN}$ and $\mathrm{zGaN}$. The first Born instability was the same across crystal structures, with wGaAs and wGaN becoming unstable when $B_{44}$ becomes nonpositive, and zGaAs and zGaN exhibiting a tetragonal shear instability [35].

\section{B. Coupled effects from pressure and temperature}

\section{Temperature effects on pressure-driven lattice instability}

Mode Grüneisen parameters, $\left\{\gamma_{i}\right\}$, defined as

$$
\gamma_{i} \equiv \frac{-V}{\omega_{i}} \frac{\partial \omega_{i}}{\partial V}
$$

give the volume sensitivity for the frequency of a phonon mode. In quantifying how phonon frequencies shift with volume, mode Grüneisen parameters provide a way to predict the onset of phonon instabilities in wGaAs and zGaAs by estimating the volumes (and thereby the pressures) at which frequencies become nonpositive (explained further in the Supplemental Material [17]). Qualitatively, Grüneisen parameters of larger magnitude indicate greater sensitivity to pressure, and faster progression towards a lattice instability. Figure 6 shows that the negative $\gamma$ become increasingly negative with pressure for both wGaAs and zGaAs at all temperatures. As pressure is applied, transverse acoustic modes at $M$ in wGaAs and at $X$ in zGaAs accelerate towards instability. This trend is reduced by temperature, however. By extrapolating phonon frequencies with volume, and using Grüneisen parameters from the sTDEP calculations, we estimate that at 0,600 , and $1200 \mathrm{~K}$, respectively, the onset of shorter wavelength phonon instabilities will occur in wGaAs at 20,27, and $30 \mathrm{GPa}$, and in zGaAs at 25, 30, and $38 \mathrm{GPa}$.

This temperature dependence is poorly described by the quasiharmonic approximation, which attributes all change in phonon frequencies to changes in volume. Using mode Grüneisen parameters from quasiharmonic phonon calculations at $1200 \mathrm{~K}$ (with $1200 \mathrm{~K}$ volumes and $0 \mathrm{~K}$ potentials), the onset of lattice stability occurs at approximately $23 \mathrm{GPa}$ in wGaAs and at $25 \mathrm{GPa}$ in zGaAs. The QHA accounts for only about $30 \%$ and $0 \%$ of the temperature-driven elevation in pressure of the lattice instabilities in wGaAs and zGaAs. Explicit anharmonicity in wGaAs and zGaAs dominates the temperature dependence of the phonon instabilities, and increases the pressure range of stability.

The Born stability criteria overestimates the pressure at which instability would occur by several GPa in both wGaAs and zGaAs, according to phonon data at $0 \mathrm{~K}$. Temperature suppresses the phonon instability, and should decrease the elastic constants. We did not calculate the temperature dependence of the elastic constants, so we do not know if the elastic instability from the Born criteria would overtake the phonon instability at temperatures below the melting temperature.

\section{Pressure effects on phonon anharmonicity}

The Grüneisen parameters of Eq. (12) change with temperature as

$$
\frac{\partial \gamma_{i}}{\partial T}=\gamma_{i}\left[\beta-\frac{1}{\omega_{i}} \frac{\partial \omega_{i}}{\partial T}\right]-\frac{V}{\omega_{i}} \frac{\partial^{2} \omega_{i}}{\partial T \partial V},
$$

so the effect of pressure on individual phonon frequencies is also temperature dependent. (Here $\beta$ is the coefficient of volume thermal expansion, but it can probably be neglected.) The second and third terms in Eq. (13) give phonon frequency shifts proportional to the product $\Delta T \times \Delta P$. [The third term in Eq. (13) is also obtained when the pure anharmonicity, proportional to $\left(\partial \omega_{i} / \partial T\right)_{V}$, has a volume dependence [41].]

If pressure and temperature were to cause additive effects on phonon frequencies, the changes of phonon frequencies and broadenings with temperature should be independent of the effects of pressure, and vice versa. This is the prediction of the quasiharmonic approximation (QHA), where all frequency shifts depend only on volume. However, Fig. 7 shows that a temperature of $1200 \mathrm{~K}$ causes shifts in the phonon spectra that differ between the QHA and the full sTDEP calculations, and furthermore, these discrepancies increase with pressure for both wGaAs and zGas. The QHA phonon spectra at $1200 \mathrm{~K}$ become more similar to $0 \mathrm{~K}$ phonon spectra at $10 \mathrm{GPa}$ than at $0 \mathrm{GPa}$. This is an effect of reduced thermal expansion at elevated pressures.

Figure 8 shows that the effects of pressure on the thermal broadening of phonon spectra are not simple. Adding $10 \mathrm{GPa}$ of pressure at $1200 \mathrm{~K}$ changes the phonon linewidths, but with mixed results; sometimes pressure increases the magnitude of the thermal broadening, but sometimes pressure decreases the phonon broadening, indicating the disappearance of previously accessible phonon decay channels. (Phonon linewidths, which are inversely proportional to phonon lifetimes, reflect the availability of opportunities for phonons to decay into combinations of lower frequency modes. The QHA does not account for broadenings of phonon spectra.) If all phonon modes had the same Grüneisen parameter, pressure would cause a proportional shift in all phonon dispersions. In this hypothetical case, the downscattering channels would remain unchanged, and the phonon anharmonicity would be unchanged by pressure. For both zGaAs and wGaAs the effect of pressure is quite different for the transverse acoustic branches and all others, which have negative and positive Grüneisen parameters, respectively. Pressure-induced changes to the phonon dispersions cause the three-phonon processes to change, thus changing the phonon anharmonicity.

Examples of how pressure alters downscattering channels are shown in Fig. 10. Pressure changes both the "source" modes, depicted as four-point stars, and the "destination" modes, the thick dots (that appear as short, thick purple and gray lines). The source modes are kept at the same $k$-vector, but as their frequencies increase with pressure, energy, and momentum conservation cause substantial changes in the allowed $k$-vectors of the destination modes. In wGaAs, a source mode is shown near $M$ along the path from $\Gamma,[0,0,0]$, to 


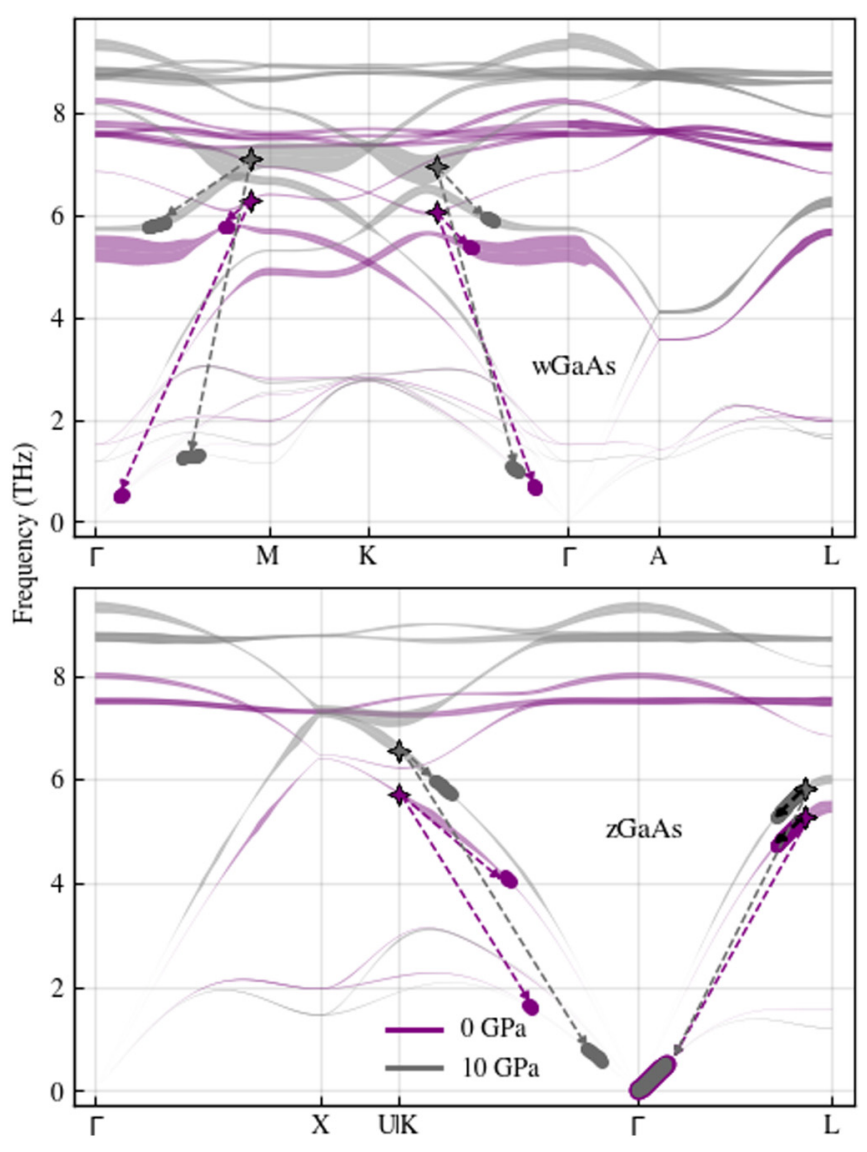

FIG. 10. Examples of decay channels that conserve crystal momenta and energy in downscattering. Pairs of source modes are marked by four-point stars, and dashed arrows point from source modes to their various decay products, shown as circular points. These modes overlay the phonon dispersions of Fig. 8. These threephonon processes are changed by pressure.

$M,\left[\frac{1}{2}, 0,0\right]$, and another near $K$ along the path from $\Gamma$ to $K,\left[\frac{2}{3}, \frac{-1}{3}, 0\right]$. Each of these source modes decays into two destination modes along the same high symmetry direction one just under $6 \mathrm{THz}$ and one near $1 \mathrm{THz}$. (There are several destination modes near $6 \mathrm{THz}$, each with a partner mode near $1 \mathrm{THz}$.) In zGaAs, longitudinal acoustic source modes are shown near $K$ along the path from $K\left(\left[\frac{3}{8}, \frac{3}{8}, \frac{3}{4}\right]\right.$ in zinc blende) to $\Gamma$, and near $L$ along the path from $\Gamma$ to $L$ at $\left[\frac{1}{2}, \frac{1}{2}, \frac{1}{2}\right]$. Each of these source modes can decay into one longitudinal acoustic mode on the same branch and one transverse acoustic mode along the same high symmetry direction, but these destination modes change significantly with pressure. On the other hand, the decay channels for the source modes in zGaAs near $L$ do not change much with pressure. The source modes have, of course, additional destination modes that lie off the high symmetry directions, but these examples are typical in that they show the mode-to-mode variations of how pressure alters the anharmonicity. More detail is presented in a table in the Supplemental Material [17].

Figure 9 shows that pressure increases the average thermal broadening in wGaAs and zGaAs at all temperatures. In contrast, for $\mathrm{wGaN}$ and $\mathrm{zGaN}$, pressure decreases the average phonon linewidth. Both wGaN and zGaN have a phonon band gap that increases with pressure. For GaN, increasing the distance between acoustic and optical modes leaves the optical modes with fewer combinations of acoustic modes into which they can decay, increasing phonon lifetimes and making GaN more harmonic with pressure, in contrast to GaAs.

\section{CONCLUSION}

The phonons and elastic properties of GaAs were calculated at simultaneous temperature and pressure using sTDEP, a fully anharmonic method, and the approximate quasiharmonic method (QHA, where all changes are attributed to effects of volume). It was found that the Born stability criteria, which implicitly account for the behavior of long wavelength phonon modes near $\Gamma$, are not the appropriate criteria for the lattice stability of either wGaAs or zGaAs because shorterwavelength phonon instabilities occurred at lower pressures. In particular, the pressure-induced softening of transverse acoustic modes at the $M$ and $X$ points in $k$-space cause lattice instabilities in wGaAs and zGaAs at 18 and $20 \mathrm{GPa}$ at $0 \mathrm{~K}$, whereas the Born stability criteria predict lattice stability until $27 \mathrm{GPa}$ in each material. Temperature causes a significant stabilization of these phonon modes, however, slowing their softening with pressure. The mode Grüneisen parameters depend significantly on temperature, causing changes in thermophysical properties that depend on the product $\Delta T \times \Delta P$.

Pressure-temperature coupling changed the anharmonic thermal broadening of phonon modes. On average for GaAs, pressure tends to increase the thermal broadening between 0 and $1200 \mathrm{~K}$. The independent shifts of phonon branches induced by pressure tend to increase the number of phononphonon interactions and phonon linewidths at $1200 \mathrm{~K}$ by creating more downscattering channels for phonon decay. In comparison to GaN with the same crystal structures, pressure had a larger effect on the average phonon broadening in GaAs than in $\mathrm{GaN}$, with $\mathrm{GaN}$ becoming more quasiharmonic with pressure, but GaAs less so. Although the Born criteria for elastic instability failed similarly in both GaAs and GaN, for $\mathrm{GaN}$ the Born instabilities occur at pressures before the onset of phonon instabilities.

\section{ACKNOWLEDGMENTS}

Work by J. E. Herriman was performed under the auspices of the US Department of Energy by Lawrence Livermore National Laboratory under Contract No. DE-AC52-07NA27344. Funding via the LLNL Livermore Graduate Scholar Program and Livermore Computing resources and support made this work possible. Effort by B.F. was supported by DOE Office of Science, BES, under Contract No. DE-FG02-03ER46055. Additional support was provided by the Capital-DOE Alliance Center, funded by the US Department of Energy through the Stewardship Sciences Academic Alliance Program. The author thanks the Quantum Simulations Group at LLNL, especially Babak Sadigh, for useful discussions. Additionally the author thanks Olle Hellman and Nina Shulumba for support of TDEP and its users. 
[1] K. Momma and F. Izumi, J. Appl. Cryst. 44, 1272 (2011).

[2] J. C. Zolper, Solid-State Electron. 42, 2153 (1998).

[3] J. Ajayan, D. Nirmal, P. Mohankumar, D. Kuriyan, A. S. A. Fletcher, L. Arivazhagan, and B. S. Kumar, Microelectron. J. 92, 104604 (2019).

[4] M. Shur, Metal semiconductor field effect transistors, The RF and Microwave Handbook, Second Edition (CRC Press, Boca Raton, FL, 2007).

[5] G. Cook, L. Billman, and R. Adcock, Photovoltaic Fundamentals (National Technical Information Service, Springfield, VA, 1995).

[6] C. del Papa, P. G. Pelfer, K. Smith, and A. Zichichi, GaAs Detectors and Electronics for High Energy Physics (World Scientific, Singapore, 1993).

[7] S. C. Cripps, RF Power Amplifiers for Wireless Communications, Second Edition (Artech House, Norwood, MA, 2006).

[8] E. Dupont, H. C. Liu, and M. Buchanan, Appl. Phys. Lett. 76, 4 (2000).

[9] R. N. Hall, G. E. Fenner, J. D. Kingsley, T. J. Soltys, and R. O. Carlson, Phys. Rev. Lett. 9, 366 (1962).

[10] B. E. Kincaid, S. Cowen, and D. Campbell, Opt. Eng. 13, 135389 (1974).

[11] C.-Y. Yeh, Z. W. Lu, S. Froyen, and A. Zunger, Phys. Rev. B 46, 10086 (1992).

[12] M. I. McMahon and R. J. Nelmes, Phys. Rev. Lett. 95, 215505 (2005).

[13] J. M. Besson, J. P. Itie, A. Polian, G. Weill, J. L. Mansot, and J. Gonzalez, Phys. Rev. B 44, 4214 (1991).

[14] S. T. Weir, Y. K. Vohra, C. A. Vanderborgh, and A. L. Ruoff, Phys. Rev. B 39, 1280 (1989).

[15] C. W. Li, X. Tang, J. A. Muñoz, J. B. Keith, S. J. Tracy, D. L. Abernathy, and B. Fultz, Phys. Rev. Lett. 107, 195504 (2011).

[16] N. Shulumba, Z. Raza, O. Hellman, E. Janzén, I. A. Abrikosov, and M. Odén, Phys. Rev. B 94, 104305 (2016).

[17] See Supplemental Material at http://link.aps.org/supplemental/ 10.1103/PhysRevB.101.214108 for additional information and data on elastic anisotropy, negative Grüneisen parameters, temperature-driven anharmonicity, pressure-induced frequency shifts, GaN comparisons, and computational details, which references other works in the bibliography [30,35,42-48]. All files related to a published paper are stored as a single deposit and assigned a Supplemental Material URL.

[18] G. Kresse and J. Furthmüller, Phys. Rev. B 54, 11169 (1996).

[19] G. Kresse and J. Furthmüller, Comput. Mater. Sci. 6, 15 (1996).

[20] W. Kohn and L. J. Sham, Phys. Rev. 140, A1133 (1965).

[21] H. J. Monkhorst and J. D. Pack, Phys. Rev. B 13, 5188 (1976).

[22] O. Hellman, I. A. Abrikosov, and S. I. Simak, Phys. Rev. B 84, 180301(R) (2011).

[23] O. Hellman and I. A. Abrikosov, Phys. Rev. B 88, 144301 (2013).
[24] O. Hellman, P. Steneteg, I. A. Abrikosov, and S. I. Simak, Phys. Rev. B 87, 104111 (2013).

[25] X. Gonze, J.-C. Charlier, D.C. Allan, and M.P. Teter, Phys. Rev. B 50, 13035 (1994).

[26] X. Gonze and C. Lee, Phys. Rev. B 55, 10355 (1997).

[27] N. Shulumba, O. Hellman, and A. J. Minnich, Phys. Rev. B 95, 014302 (2017).

[28] D. West and S. K. Estreicher, Phys. Rev. Lett. 96, 115504 (2006).

[29] I. Errea, M. Calandra, and F. Mauri, Phys. Rev. B 89, 064302 (2014).

[30] D. S. Kim, O. Hellman, J. Herriman, H. L. Smith, J. Y. Y. Lin, N. Shulumba, J. L. Niedziela, C. W. Li, D. L. Abernathy, and B. Fultz, Proc. Ntal. Acad. Sci. 115, 1992 (2018).

[31] J. Wang, S. Yip, S. R. Phillpot, and D. Wolf, Phys. Rev. Lett. 71, 4182 (1993).

[32] J. Wang, J. Li, S. Yip, S. Phillpot, and D. Wolf, Phys. Rev. B 52, 12627 (1995).

[33] B. B. Karki, G. J. Ackland, and J. Crain, J. Phys.: Condens. Matter 9, 8579 (1997).

[34] G. V. Sin'ko and N. A. Smirnov, J. Phys.: Condens. Matter 14, 6989 (2002).

[35] J. E. Herriman, O. Hellman, and B. Fultz, Phys. Rev. B 98, 214105 (2018).

[36] G. Venkataraman, L. Feldkamp, and V. Sahni, Dynamics of Perfect Crystals (MIT Press, Cambridge, MA, 1975).

[37] L. Mauger, J. E. Herriman, O. Hellman, S. J. Tracy, M. S. Lucas, J. A. Muñoz, Y. Xiao, J. Li, and B. Fultz, Phys. Rev. B 95, 024308 (2017).

[38] D. M. Clatterbuck, C. R. Krenn, M. L. Cohen, and J. W. Morris, Phys. Rev. Lett. 91, 135501 (2003).

[39] G. Grimvall, B. Magyari-Köpe, V. Ozolins, and K. A. Persson, Rev. Mod. Phys. 84, 945 (2012).

[40] A. G. Lyapin and V. V. Brazhkin, Phys. Rev. B 54, 12036 (1996).

[41] X. Tong, X. Xu, B. Fultz, H. Zhang, T. A. Strobel, and D. Y. Kim, Phys. Rev. B 95, 094306 (2017).

[42] S. I. Ranganathan and M. Ostoja-Starzewski, Phys. Rev. Lett. 101, 055504 (2008).

[43] R. Hill, Proc. Phys. Soc. Section A 65, 349 (1952).

[44] S. I. Novikova, Sov. Phys. Solid State 3, 129 (1961).

[45] A. Jain, S. P. Ong, G. Hautier, W. Chen, W. D. Richards, S. Dacek, S. Cholia, D. Gunter, D. Skinner, G. Ceder, and K. A. Persson, APL Mater. 1, 011002 (2013).

[46] H. J. McSkimin, A. Jayaraman, and P. Andreatch, J. Appl. Phys. 38, 2362 (1967).

[47] H.-L. Cui, F. Luo, X.-R. Chen, and G.-F. Ji, Can. J. Phys. 87, 153 (2009).

[48] C. W. Garland and K. C. Park, J. Appl. Phys. 33, 759 (1962). 